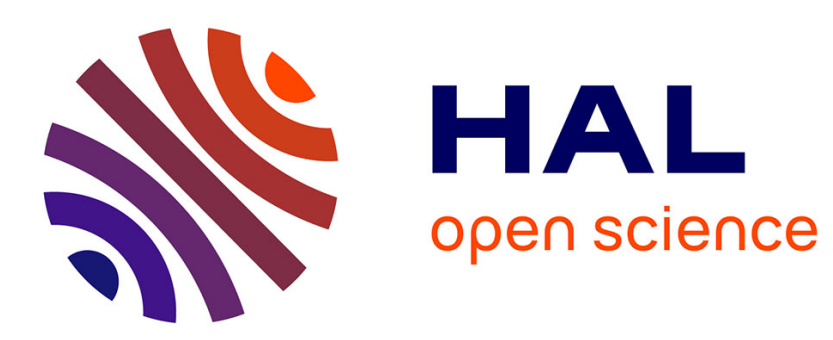

\title{
Apports potentiels de la théorie des possibilités à l'évaluation des risques environnementaux
}

Bernard Côme, Dominique Guyonnet, Samir Magnouni

\section{To cite this version:}

Bernard Côme, Dominique Guyonnet, Samir Magnouni. Apports potentiels de la théorie des possibilités à l'évaluation des risques environnementaux. Environnement, Ingénierie \& Développement, 1997, Nº - 3ème Trimestre 1997, pp.40-46. 10.4267/dechets-sciences-techniques.806 . hal-03182891

\section{HAL Id: hal-03182891 https://hal.science/hal-03182891}

Submitted on 26 Mar 2021

HAL is a multi-disciplinary open access archive for the deposit and dissemination of scientific research documents, whether they are published or not. The documents may come from teaching and research institutions in France or abroad, or from public or private research centers.
L'archive ouverte pluridisciplinaire HAL, est destinée au dépôt et à la diffusion de documents scientifiques de niveau recherche, publiés ou non, émanant des établissements d'enseignement et de recherche français ou étrangers, des laboratoires publics ou privés.

\section{(c)(1)}

Distributed under a Creative Commons Attribution| 4.0 International License 


\section{APPORTS POTENTIELS DE LA THÉORIE DES POSSIBILITÉS À L'ÉVALUATION DES RISQUES ENVIRONNEMENTAUX}

Bernard Côme, Dominique Guyonnet, Samir Magnouni*

Antea

Certains problèmes environnementaux, tel l'impact potentiel d'un site pollué sur les milieux vivants, se caractérisent initialement par le petit nombre de données descriptives du problème, et l'incertitude sur les critères d'acceptabilité des éventuels relargages de polluants. L'article expose comment les outils de la théorie des possibilités, en particulier la notion de nombre flou et celle de mesure de nécessité (et de possibilité) permettent d'évaluer l'acceptabilité (ou non) d'une telle situation. On en conclut que cet ensemble de concepts constitue un mode de mise en œuvre raisonnable du " principe de précaution », évitant toute surenchère excessivement sécuritaire. Ces outils relativement nouveaux gagneraient sans doute à être mieux connus des décideurs (organismes à l'origine des réglementations, administrations...).

Some environmental problems, such as the impact of a contaminated site, are characterized by the initial paucity of reliable data, and the uncertainty about acceptance criteria for released pollutants. It is shown how the " theory of possibility ", particularly the concepts of fuzzy numbers and of necessity measure, can be used to assess the acceptability of such situations. This theory is advocated to supply an acceptable implementation scheme for the " principle of precaution ", by avoiding overconservative assumptions. These rather new tools would deserve improved communication mainly toward regulators and decisionmakers.

\section{INTRODUCTION}

L'évaluation des risques liés aux sites pollués, par exemple celle des conséquences du relargage de polluants émis sur les êtres vivants du voisinage, se heurte souvent à la difficulté de collecter suffisamment de données descriptives du site pour garantir une précision acceptable du résultat. Les grandeurs descriptives du système envisagé (la source de polluants : nature, toxicité ; leurs modes de transfert, notamment par l'écoulement des eaux souterraines et/ou de surface; les éventuels récepteurs de la pollution ainsi transportée : population, faune, flore), sont inévitablement affectées d'une incertitude pouvant résulter de la difficulté de réaliser des mesures, prélèvements, analyses, en nombre suffisant.

Un remède à cette situation consiste, bien entendu, à développer les méthodes d'investigation rapides et peu coûteuses permettant d'acquérir un grand nombre de mesures, en vue d'un traitement statistique approprié de ces dernières. Le calcul des risques pourra alors faire appel aux simulations dites de « Monte-Carlo », où les données d'entrées des calculs sont échantillonnées à partir de leur densité de probabilité, de manière à obtenir un histogramme et/ou une fonction de répartition (probabiliste) des conséquences (Vose, 1996).

L'usage de telles méthodes probabilistes se révèle cependant délicat, en particulier aux premiers stades de l'investigation d'un site pollué, où une évaluation précoce des risques serait néanmoins souhaitable, de manière à orienter la campagne de reconnaissance et les éventuelles mesures curatives à mettre en œuvre. Le petit nombre de mesures disponibles est alors un obstacle à l'élaboration des densités de probabilité (ou fonctions de répartition) pour les données des calculs. Par contre, un certain « degré de confiance " peut être affecté aux quelques valeurs mesurées, éventuellement complétées par des éléments plus subjectifs tels des opinions d'experts, des données provenant de situations analogues... Il est alors souhaitable de disposer d'un cadre conceptuel, et d'outils de calculs, adaptés pour la prise en compte de telles données imprécises ou vagues.

\section{ÉLÉMENTS DE LA THÉORIE DES POSSIBILITÉS, APPLICABLES À L'ÉVALUATION DES RISQUES}

\section{Généralités - Notions d'ensembles et de nombres flous}

La notion d'ensembles flous a été élaborée par $L$. Zadeh aux États-Unis dans les années 1960, pour fournir entre autres un mode de traitement de connaissances imprécises ou vagues (incl. les aspects semi-qualitatifs) moins exigeant, sur le plan de l'axiomatique, que la théorie des probabilités (Zadeh, 1965). 
La théorie permet de définir en particulier les concepts de nombre précis (anglais : crisp) et de nombre flou (anglais : fuzzy). Pour ce dernier concept, on peut considérer par exemple l'estimation de la taille d'un individu $(1,7 \mathrm{~m})$, et quantifier la plus ou moins grande « ressemblance » d'une évaluation de cette taille avec la valeur I,7 $\mathrm{m}$. II est possible ainsi de définir une fonction d'appartenance, $\mu$, traduisant la « ressemblance » d'un nombre $n$ donné avec le nombre I,7; par convention, elle vaudra I lorsque $n$ sera exactement égal à $I, 7$, pour décroître à zéro pour $n \leq 1,5$ et $n \geq 1,9$ par exemple, les valeurs intermédiaires étant décrites par une fonction continue. Cette fonction d'appartenance traduit alors la notion qualitative de « degré de confiance » évoquée plus haut. Elle ne doit pas, bien sûr, être confondue avec une représentation statistique de la répartition des tailles d'individus.

La figure I représente le nombre flou correspondant, muni de sa fonction d'appartenance $\mu$. Cette représentation inclut donc explicitement l'aspect imprécis et/ou vague des connaissances.

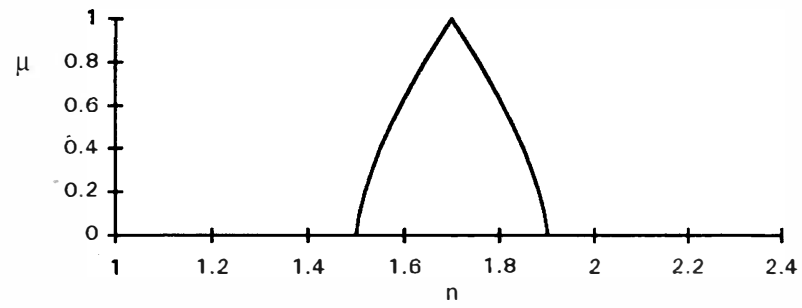

Figure I : Le nombre flou correspondant à une taille d'individu évaluée à environ $1,7 \mathrm{~m}$

A cette notion de nombres flous et d'ensembles flous est associé un cadre mathématique connu sous le nom de « théorie des possibilités », permettant de construire en particulier une logique floue généralisant la logique booléenne du « oui ou non », en introduisant le « peut-être » et les moyens de le quantifier. Ces concepts ont été développés en France, notamment à l'Université de Toulouse (Prade et Dubois, 1988), et récemment examinés dans une optique industrielle (Club Crin Logique Floue, 1997).

Ces théories possèdent dès maintenant des applications en hydrogéologie (Kindler et al., 1988 ; Dou et al., 1995). On se propose ici de présenter ces outils en vue de l'évaluation précoce des risques liés aux sites pollués, avec prise en compte de l'imprécision inhérente à toute évaluation de ce type ; l'objectif étant de pouvoir, avec un minimum de connaissances de la situation (et éventuellement des données plutôt qualitatives ou peu quantifiées), d'avoir une mesure raisonnable de l'acceptabilité (ou non) du risque.

On adoptera dans ce but le schéma traditionnel de l'évaluation d'un site pollué, impliquant une certaine connaissance de la source de pollution, des vecteurs de cette dernière, et des cibles éventuellement affectées. Ces composants interviennent dans un modèle (mathématique) du système. La résolution du problème suppose que soient mis au point un cadre conceptuel et un outil de calcul satisfaisants pour chacun des quatre aspects ci-dessous :
- représentation de données de calcul sous forme de nombres flous :

- réalisation de calculs (arithmétique, algèbre) en nombres flous ;

- représentation de critères d'acceptabilité sous forme floue; - mode de comparaison d'un résultat de calcul flou à un critère flou, et obtention d'un indice quantifiant cette comparaison.

\section{Représentation de grandeurs imprécises} sous forme de nombres flous, en vue de leur utilisation ultérieure dans des calculs

Les connaissances (mesures éventuellement disponibles, jugements de spécialistes, estimations...) sont à utiliser pour définir ce qu'on appelle le noyau et le support d'un nombre flou (figure 2).

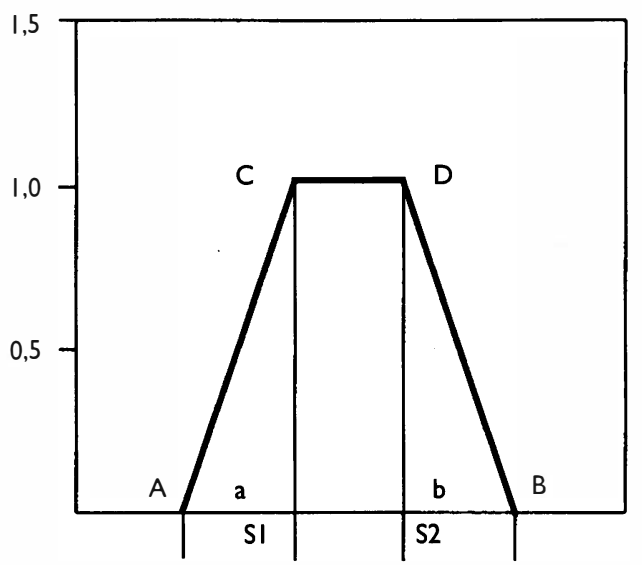

Figure 2 : Noyau et support d'un nombre flou

Le noyau correspond aux valeurs mesurées ou estimées les plus plausibles $(\mu=1)$, tandis qu'à l'extérieur du support se trouvent les valeurs impossibles $(\mu=0)$. Les nombres $a$ et $b$ sont appelés étalement (resp. à gauche et à droite).

La figure 2 peut se traduire numériquement de deux façons principales, correspondant à deux modes d'expression des nombres flous, permettant de joindre les points de fonction d'appartenance 0 à ceux de fonction d'appartenance I, soit $A$ à $C$, et $D$ à $B$ :

- Sous forme de représentation dite « $L-R$ » : il s'agit de se donner une fonction décrivant les courbes passant par ces couples de points, une pour la partie gauche $A-C(L=l e f t)$, une pour la partie droite $D-B(R=$ right). On montre que des fonctions du type $1-x^{p}$, avec $p$ paramètre de forme, conviennent pour la majorité des cas. La simplification $p=1$ fournit des représentations sous forme de droites; on obtient ainsi un nombre flou sous forme de trapèze, qu'on peut noter simplement $\left(S_{1}, S_{2}, a, b\right)$ en faisant intervenir support et étalements. $\mathrm{Si}_{1} \mathrm{~S}_{1}=\mathrm{S}_{2}$, on définit un nombre flou triangulaire.

- Sous forme de coupes de niveau $\alpha$, ou « $\alpha$-coupes » (figure 3). II s'agit, pour chaque valeur $\alpha$ de la fonction d'appartenance relative au nombre flou étudié, de se donner la gamme des valeurs envisageables pour ce nombre. Partant 


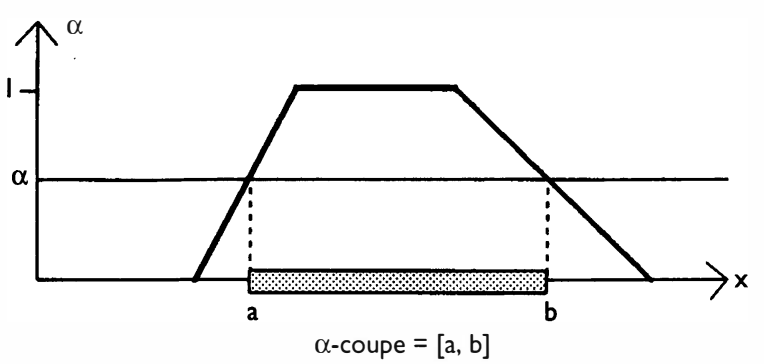

Figure 3 : Coupe de niveau

de $\mu=0$ et progressant jusqu'à $\mu=1$, on peut alors « superposer » des sections horizontales et reconstituer ainsi l'intervalle ou le nombre flou.

On notera qu'il ne semble pas encore exister de règle uniformément acceptée pour effectivement construire des nombres flous à partir de connaissances disponibles.

\section{Réalisation de calculs en nombres flous}

Des axiomes généraux de la théorie des ensembles flous, dont le " principe d'extension », permettent de définir les règles d'une arithmétique et d'une algèbre en nombres flous; les formules conduisent cependant à des calculs assez lourds. Une méthode plus simple et fournissant une bonne approximation des résultats est celle dite " par découpage " en utilisant les $\alpha$-coupes décrites plus haut. On montre en effet que, si f est une fonction continue, on a généralement pour 2 nombres flous $P$ et $Q$ :

$[f(P, Q)]_{\alpha}=f\left([P]_{\alpha},[Q]_{\alpha}\right)$

où $[P]_{\alpha}$ représente par exemple l' $\alpha$-coupe de $P$.

On peut donc obtenir les $\alpha$-coupes d'un résultat de calcul à partir des $\alpha$-coupes des données d'entrée.

Pratiquement, la coupe de niveau $\alpha$ d'un résultat de calcul $f(P, Q)$ peut s'obtenir comme suit :

I - se donner une coupe de niveau $\alpha$ de $P$ et de $Q$,

2 - sélectionner dans chacune une valeur, soit $P_{1}$ et $Q_{1}$,

3 - calculer $f\left(P_{1}, Q_{1}\right)$,

4 - recommencer un certain nombre $n$ de fois les opérations 2 et 3 ci-dessus,

5 - dans l'ensemble des $n$ valeurs de $f(P, Q)$ ainsi obtenues, identifier la valeur minimale et la valeur maximale,

6 - affecter ces valeurs respectivement aux limites inférieure et supérieure de la coupe de niveau $\alpha$ de $f(P, Q)$, 7 - répéter l'ensemble des opérations I à 6 ci-dessus pour une nouvelle valeur de $\alpha$,

8 - reconstituer ainsi le résultat flou $f(P, Q)$ par ses coupes de niveau $\alpha$.

Représentation de critères d'acceptabilité sous forme de nombres flous

Une manière simple de traduire un seuil d'acceptabilité imprécis consiste à attribuer la valeur I à la fonction d'appartenance des valeurs totalement acceptables, et 0 pour les valeurs inacceptables, l'imprécision étant maté-

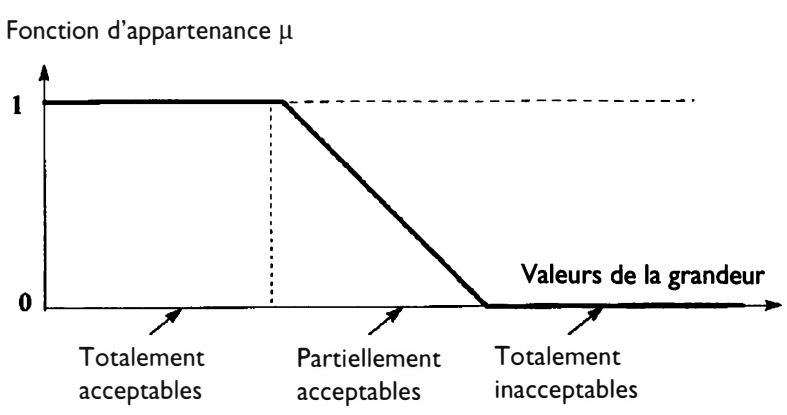

Gammes de valeurs

Figure 4 : Forme simple d'un seuil d'acceptabilité flou

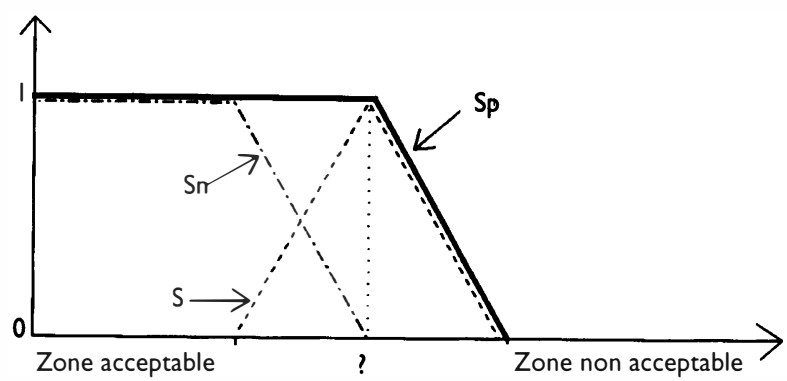

Figure 5 : Sous-ensembles Sp et Sn

rialisée par une décroissance progressive de la fonction d'appartenance (figure 4).

Si le seuil d'acceptabilité est représenté non plus par une gamme de valeurs mais par un nombre flou $S$ de fonction d'appartenance $\mu_{\mathrm{s}}$ (par exemple, un nombre triangulaire), on est conduit à définir deux sous-ensembles, introduits par la théorie des possibilités (Prade et Dubois, 1988), et illustrés par la figure 5 :

- le sous-ensemble flou des nombres qui peuvent être inférieurs à $S$, qu'on notera $S_{p}$; sa fonction d'appartenance $\mu_{\text {sp }}$ est définie pour tout $\omega$ par :

$$
\begin{gathered}
\forall \omega, \mu_{\mathrm{sp}}(\omega)=\operatorname{Sup}\left[\mu_{\mathrm{s}}(\mathrm{u})\right] \\
\mathrm{u} \geq \omega
\end{gathered}
$$

où Sup (supremum) désigne la plus grande des valeurs prises par $\mu \mathrm{s}$.

- le sous-ensemble flou des nombres qui sont nécessairement inférieurs à $S$, noté $S_{n}$; la fonction d'appartenance $\mu s_{n}$ est définie pour tout $\omega$ par :

$$
\begin{aligned}
\forall \omega, \mu_{\mathrm{s}}(\omega) & =\operatorname{lnf}\left[1-\mu_{\mathrm{s}}(\mathrm{u})\right] \\
\mathrm{u} & \leq \omega
\end{aligned}
$$

où Inf (infimum) désigne la plus petite des valeurs prises par (I- $\mu \mathrm{s})$.

Il est donc possible, à l'aide de $S_{p}$ et $S_{n}$, de définir un mode d'acceptabilité « tolérant » ou « strict ».

Comparaison d'un nombre flou à un critère d'acceptabilité flou, et identification d'un indicateur de cette comparaison

La figure 6 visualise le problème ainsi posé : « la grandeur floue $R$ est-elle inférieure au seuil flou $S$ ? » 
La théorie des possibilités permet d'attribuer deux mesures à la proposition « $R$ est inférieur à $S$ », à savoir :

- une mesure de possibilité $\Pi(0 \leq \Pi \leq 1)$ de cette proposition : si $\mu_{R}$ et $\mu_{S}$ sont les fonctions d'appartenance respectivement du résultat et du seuil, on la définit par :

$\Pi(R \leq S)=\operatorname{Sup} \min \left[\mu_{R}(u), \mu_{S}(u)\right]$

$\mathrm{u}$

où min (minimum) est la plus petite des deux valeurs $\mu_{R}$ et $\mu_{\mathrm{s}}$ pour $u$ donné, et Sup est comme défini plus haut.

- une mesure de nécessité $N(0 \leq N \leq 1)$ de cette proposition ; avec la même notation, on posera :

$$
N(R \leq S)=\operatorname{lnf}_{u} \max \left[\left(1-\mu_{R}(u), \mu_{S}(u)\right]\right.
$$

où max (maximum) est la plus grande des deux valeurs $\left(I-\mu_{R}\right)$ et $\mu_{s}$ pour $u$ donné, et Inf est comme défini plus haut.

Les valeurs correspondantes de $\prod$ et de $\mathrm{N}$ sont repérées sur la figure 7 pour un nombre flou $R$ triangulaire. Dans le cas général, $\Pi$ et $N$ peuvent se calculer à l'aide de quelques lignes de code informatique, à partir de leur définition.

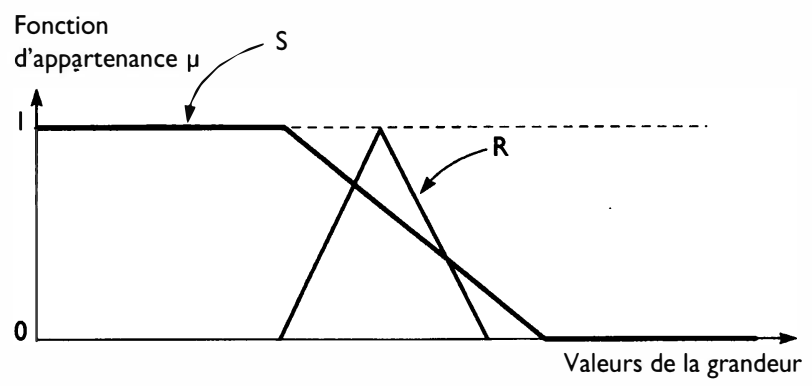

Figure 6 : Comparaison d'un résultat à un seuil d'acceptabilité

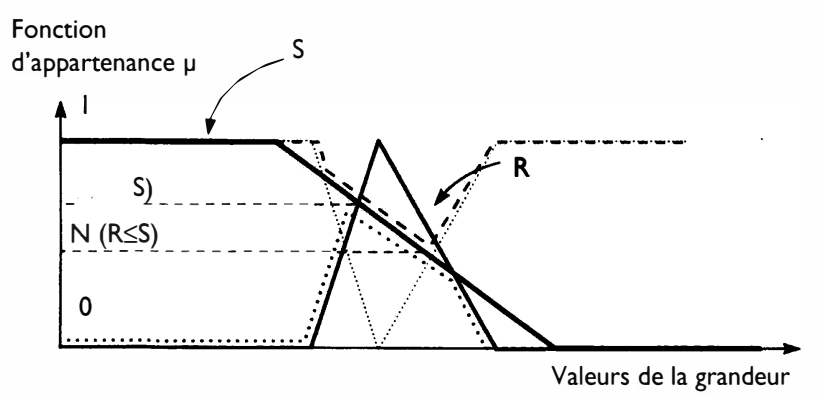

- Mesure de possibilité : $\Pi(R \leq S): \operatorname{Sup}\left\{\operatorname{Min}\left[\mu_{r}(u), \mu_{s}(u)\right]\right\}$

- Mesure de nécessité : $N(R \leq S)=\operatorname{lnf}\left\{\operatorname{Max}\left[1-\mu_{r}(u), \mu s(u)\right]\right\}$

Figure 7 : Mesures de nécessité et de possibilité de R S

On remarquera que ces mesures généralisent la logique booléenne faisant appel aux notions de possibilité et de nécessité ; dans ce dernier cadre, si une proposition $A$ est nécessaire $\left(N_{(A)}=1\right)$, alors son contraire est impossible $\left(\prod_{(\bar{A})}=0\right)$, si $\bar{A}$ représente le contraire de $A$. Plus généralement, on a en logique floue : $N(A)=1-\prod(\bar{A})$.

La mesure de nécessité $N$ de $R \leq S$ (attitude « stricte ») est inférieure à la mesure de possibilité $\Pi$ de $R \leq S$ (attitude plus tolérante). Dans une communication personnelle, Prade et Dubois (1995) conseillent de sélectionner $\mathrm{N}$ comme indicateur traduisant le mieux une attitude dite « d'aversion au risque ». En particulier, si $N=0$ et $\Pi=0$, la situation est inacceptable; le seuil est dépassé. Si $N=0$ et $\Pi \neq 0$, la situation n'est vraisemblablement pas acceptable; il s'agit de compléter l'information. Si $\mathrm{N}>0$, alors la situation est acceptable à $\mathrm{N} \%(\mathrm{~N}=$ I représentant l'acceptabilité à $100 \%)$.

Dans le cas général où le seuil $S$ est défini par un nombre flou ordinaire, on détermine les deux sous-ensembles flous $S_{n}$ et $S_{p}$ présentés plus haut, et on calcule 4 indices:

- les mesures de possibilité (PP) et de nécessité (NP) que $R$ soit inférieur à $S_{p}$;

- les mesures de possibilité (PN) et de nécessité (NN) que $R$ soit inférieur à $S_{n}$.

En fonction du problème à traiter, on choisira un ou une combinaison de ces 4 indices pour juger de l'acceptabilité de la situation, en donnant une préférence à l'indice $\mathrm{NN}$.

\section{EXEMPLE D'APPLICATION}

Le cas d'un site pollué hypothétique, et de l'évaluation de l'acceptabilité (ou non) des risques qu'il peut entraîner, va maintenant servir à illustrer l'emploi des concepts et outils de la théorie des possibilités.

\section{Calcul de transfert en nombres flous}

On présente ici un exemple simple, susceptible d'une solution par voie analytique. Pour des problèmes plus complexes, des approches numériques sont d'ores et déjà envisageables (Bardossy et al., 1995).

Dans le schéma simplifié de la figure $8 \mathrm{a}$, un site de dimension $L_{1}$ dans la direction d'écoulement des eaux souterraines dans un aquifère sous-jacent, et $L_{2}$ dans la direction perpendiculaire à ce même écoulement, est contaminé par un polluant organique qui imprègne les terrains superficiels en phase résiduelle. Une pluie efficace $P_{\text {eff }}$ est à l'origine d'une mobilisation de polluants dissouts à une concentration supposée constante $C_{\circ}$ (prise par exemple égale à la limite de solubilité du polluant considéré). Une dilution par de l'eau souterraine non-contaminée, à débit spécifique $q_{a q}$, a lieu sur une épaisseur $L_{3}$ dans l'aquifère sous-jacent, directement à l'aplomb de la zone polluée. Dans une optique sécuritaire on se place en régime permanent pour lequel on obtient un « plateau » de concentration à l'aplomb du site, de valeur $C_{d}$, qui est fonction du rapport des flux d'eau polluée et propre. On considère ensuite la migration de cette concentration $C_{d}$ dans l'aquifère, vers un point-cible situé à une certaine distance à l'aval hydraulique de la zone polluée. Seule la dispersion est prise en compte en tant que mécanisme d'atténuation de la pollution vers l'aval hydraulique.

L'équation aux dérivées partielles gouvernant l'évolution spatiale de la concentration $C$ dans l'aquifère en régime permanent est (en négligeant la dispersion verticale-transverse) :

$D_{x} \frac{\partial^{2} C}{\partial x^{2}}+D_{y} \frac{\partial^{2} C}{\partial y^{2}}-v \frac{\partial C}{\partial x}=0$ 


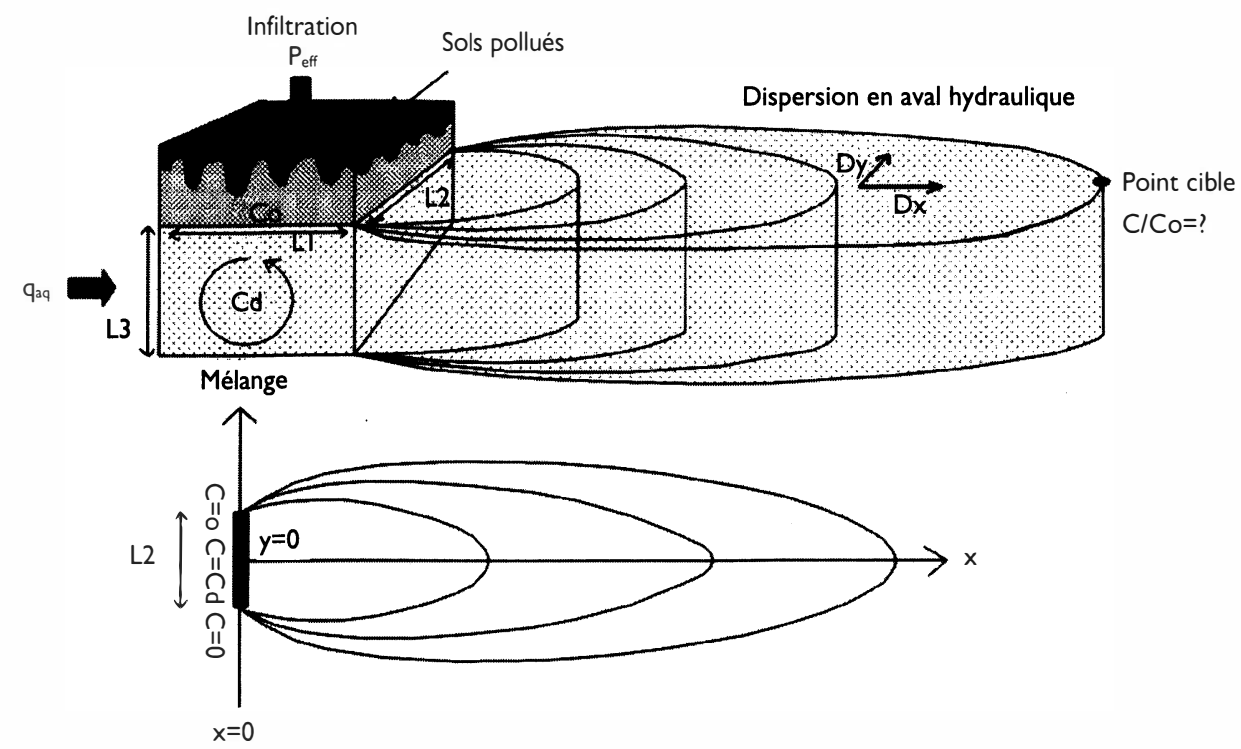

Figure 8 a et $b$ : Modèle simplifié d'une pollution émise par un site pollué

avec comme conditions aux limites (voir la figure 8b) :

$C(x=0, y)=C_{d}$ pour $-\frac{L_{2}}{2}<y<\frac{L_{2}}{2}$

$C(x=0, y)=0$ pour $y<-\frac{L_{2}}{2}$ ou $y>\frac{L_{2}}{2}(8)$

$C(x, y= \pm \infty)=C(x, y= \pm \infty)=0$

où :

$C$ est la concentration dans l'aquifère au temps t et aux coordonnées $(x, y)$ (voir figure 8b),

$D_{x}$ est la composante en $x$ du coefficient de dispersion, et $D_{y}$ sa composante en $y$,

$\checkmark$ est la vitesse de l'eau dans l'aquifère,

et $C_{d}$ est la concentration dans l'aquifère à l'aval immédiat du site pollué.

Le coefficient de dispersion, $D$, intègre à la fois la dispersion mécanique et la diffusion moléculaire (voir par exemple Freeze et Cherry, 1979). Toutefois, dans un aquifère perméable comme celui considéré plus bas, la composante diffusionnelle est négligeable. Le coefficient de dispersion s'écrit alors :

$D_{x}=\alpha_{x} v$ et $D_{y}=\alpha_{y} v$,

où $\alpha_{x}$ et $\alpha_{y}$ sont les dispersivités en $x$ et en $y$.

Il est rappelé que la vitesse de l'eau dans l'aquifère est liée au débit spécifique $q_{a q} p a r$ :

$\mathrm{v}=\frac{\mathrm{q}_{\mathrm{aq}}}{\mathrm{n}}$

où $n$ est la porosité de l'aquifère. Le débit spécifique $q_{\text {aq }}$ est lié à la perméabilité (K) du milieu aquifère, et au gradient hydraulique (i) par la loi de Darcy : $q_{\text {aq }}=-\mathrm{Ki}$.

Aucune barrière minérale peu perméable n'étant située entre les terrains pollués et le milieu aquifère, la contribution dispersive du flux polluant provenant de ces terrains sera négligée. Le flux polluant sera contrôlé par la pluie efficace, et la concentration après dilution dans l'aquifère $\left(C_{d}\right)$ peut s'écrire :

$$
C d=\frac{P_{\text {eff }} L_{1} C_{0}}{P_{\text {eff }} L_{1}+q_{a q} L_{3}}
$$

On notera que la non-saturation du milieu situé entre les terrains pollués et l'aquifère aura principalement pour effet de modifier la vitesse de transfert du flux polluant. Par contre la quantité de masse émise en régime stabilisé sera sensiblement la même. A noter également que l'épaisseur $L_{3}$ ne représente pas nécessairement l'épaisseur de l'aquifère mais plutôt celle d'une « couche de mélange » pour laquelle on estime une concentration moyenne dans l'aquifère à l'aplomb du site.

La solution de l'équation (6), soumise aux conditions décrites par les équations (7) à $(10)$ est (modifiée d'après Cleary et Ungs, 1978) (II) :

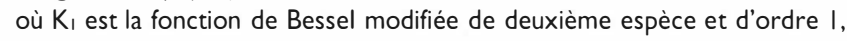

$\frac{C(x, y)}{C_{0}}=\frac{P_{e f f} L_{1}}{P_{e f f} L_{1}+q_{a q} L_{3}} \cdot \frac{1}{\pi \sqrt{D_{x} D_{y}}} \exp \left[\frac{v x}{2 D_{x}}\right] \cdot \int_{\mathrm{r}=-L 2 / 2}^{\tau=L 2 / 2} \frac{\sqrt{\frac{v^{2}}{4 D_{x}}}}{\sqrt{\frac{1}{D_{x}}+\frac{(y-\tau)^{2}}{D_{y} x^{2}}}} K_{1}\left[\sqrt{\frac{v^{2}}{4 D_{x}}\left(\frac{x^{2}}{D_{x}}+\frac{(y-\tau)^{2}}{D_{y}}\right)}\right] d \tau$

et $\tau$ est la variable d'intégration.

Dans ce qui suit, des nombres flous sont utilisés pour traduire l'imprécision sur les grandeurs telles la perméabilité du milieu aquifère, l'épaisseur de la zone de mélange, et la dispersivité, grandeurs dont l'acquisition sur le terrain, ou l'estimation a priori, sont toujours problématiques.

La figure 9 rassemble les représentations adoptées pour les nombre flous $K, L_{3}, \alpha_{x}$ et $\alpha_{y}$. L'aquifère est constitué de sables perméables de perméabilité « environ » $10^{-3} \mathrm{~m} / \mathrm{s}$. Le support de ce nombre flou s'étale sur un ordre de grandeur, entre $5^{*} 10^{-4}$ et $5^{*} 10^{-3} \mathrm{~m} / \mathrm{s}$. L'épaisseur de la couche de mélange est d'environ $20 \mathrm{~m}$, tandis que les valeurs de la dispersivité en $x$ et en $y$ sont respectivement d'environ 20 et $5 \mathrm{~m}$.

Les autres valeurs de paramètres sont supposées de type « crisp » (non floues). La pluie efficace est de 200 mm/an, la lar- 

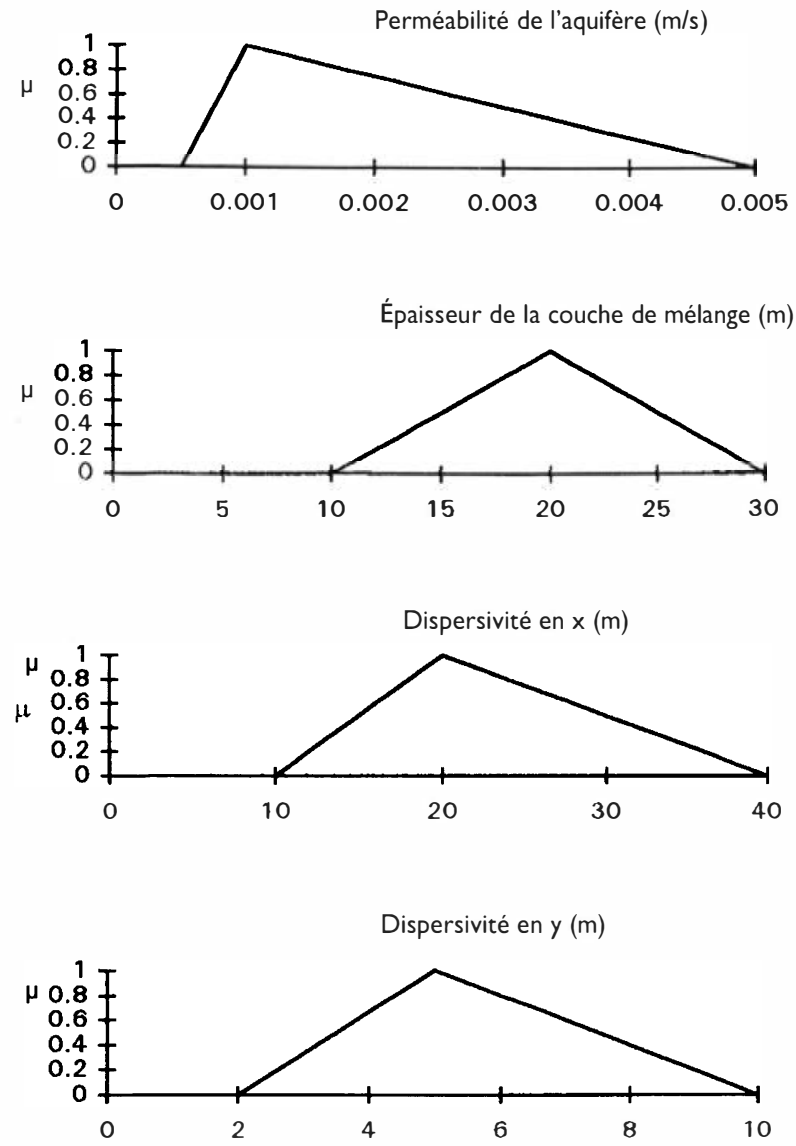

Figure 9 : Représentations floues pour la perméabilité (K), l'épaisseur de la couche de mélange (L3), et les composantes en $x$ et $y$ de la dispersivité ( $x$ et $y$ )

geur de la zone polluée $\left(L_{1}\right)$ est de $50 \mathrm{~m}$, la longueur $L_{2}$ est de $25 \mathrm{~m}$, la porosité de l'aquifère est de $20 \%$ et le gradient hydraulique dans l'aquifère est de $0,5 \%$.

Le résultat du calcul flou effectué par la méthode de découpage présentée précédemment, à partir de l'équation (II), pour un point-cible situé à $200 \mathrm{~m}$ en aval hydraulique de la zone polluée, est présenté en figure 10 . On obtient une concentration "réduite " $(\mathrm{C} / \mathrm{Co})$ la plus vraisemblable égale à $7,4 * 10^{-4}$, tandis que le support de ce nombre flou

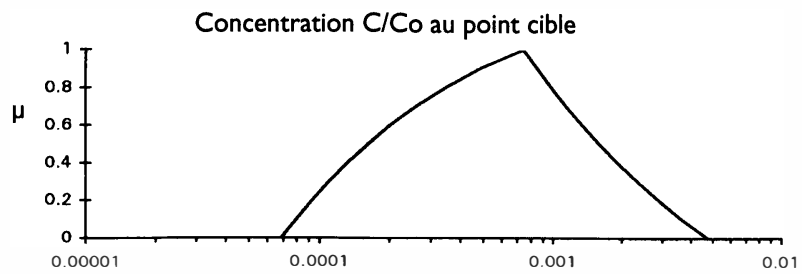

Figure 10 : Concentration floue calculée pour un point cible situé à $200 \mathrm{~m}$ en aval hydraulique de la zone polluée s'étale entre $6,9 * 10^{-5}$ et $4,8 * 10^{-3}$.

II convient de noter ici que si le calcul en nombres flous (« fuzzification ») de la formule (II) n'offre pas de difficultés particulières, la résolution complète de l'équation aux dérivées partielles (6) avec des paramètres flous pose encore un certain nombre de problèmes théoriques (Shaw, 1989).

\section{Principe d'évaluation de risque en nombres flous}

En matière d'évaluation de risque, une concentration $C$ floue calculée de cette manière, pour un polluant spécifique, peut être assimilée à la «PEC » (Predicted Environmental Concentration), mentionnée dans le Règlement communautaire $n^{\circ}$ 1488/94 du 28 juin 1994 concernant l'évaluation des risques liés aux substances toxiques existantes (Commission Européenne, 1994). II s'agit maintenant de la comparer à la "PNEC " (Predicted No Effect Concentration), de manière à caractériser le risque correspondant au polluant considéré. Une PNEC floue pourrait se construire comme présenté sur la figure II, à partir des concepts développés en particulier dans le « Technical Guidance Document » sur la mise en œuvre du Règlement communautaire de 1994 (Commission Européenne, 1996). La comparaison de la PEC à cette PNEC, pour le polluant considéré, se ferait alors en calculant les mesures de nécessité et de possibilité correspondantes ; l'acceptabilité étant, comme indiqué précédemment, quantifiée de préférence par la mesure de nécessité obtenue.

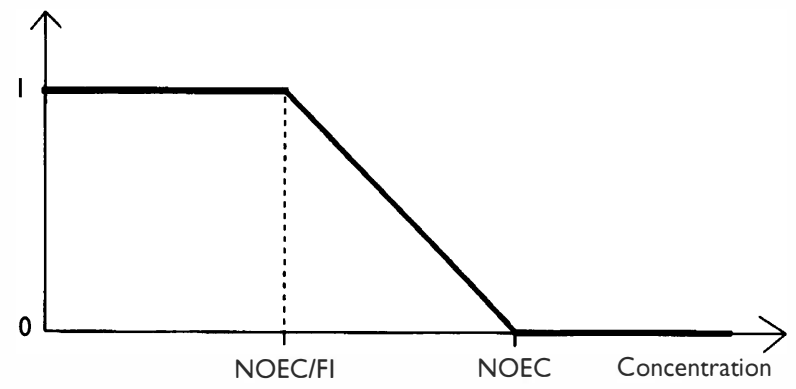

Figure II : Représentation envisageable pour une PNEC floue (NOEC est la concentration sans effets observés pour le polluant ; FI est un facteur d'incertitude pouvant valoir jusqu'à 1000)

On notera que le choix de la mesure de nécessité de la proposition «PEC PNEC », correspondant à une optique d'acceptabilité stricte, serait compatible avec une application « raisonnable » du principe de précaution; cette mesure de nécessité est en effet appliquée à des grandeurs ne résultant pas elles-mêmes de l'emploi systématique de valeurs sécuritaires (majorantes) pour les données d'entrée des calculs. Bien entendu, seule une autorité compétente pourra fixer la valeur de la mesure de nécessité à partir de laquelle la PEC sera déclarée acceptable par rapport à la PNEC, dans le cadre de la théorie des possibilités. 


\section{CONCLUSIONS ET PERSPECTIVES}

La théorie des possibilités fournit des concepts appropriés pour l'évaluation des risques liés aux sites pollués ou à des décharges défectueuses. En outre, la mise en œuvre de ces concepts sous forme de calculs ne pose pas de difficultés rédhibitoires.

Les notions de mesures de nécessité et de possibilité, relatives à l'acceptabilité de résultats par rapport à des seuils prédéterminés, semblent constituer une étape vers une application raisonnable du principe de précaution, permettant d'apprécier au cas par cas des situations spécifiques, et de faciliter le rôle final du décideur.

II n'en demeure pas moins que ces notions et outils n'ont pas encore atteint la popularité, ni la maturité, des techniques d'analyse de type probabiliste, bien que les limitations de ces dernières soient par contre de mieux en mieux cernées (Shaw, 1989). Cette situation pourra bien sûr évoluer au fur et à mesure de la réalisation de ce type d'analyse sur des cas réels, et de la présentation des résultats aux organismes chargés d'élaborer les réglementations, et aux décideurs (administrations, etc.).

\section{* Bernard Côme, Dominique Guyonnet, Samir Magnouni}

Antea - 3, avenue Claude Guillemin - BP 6119 - 45061 Orléans cedex 02

Remerciements

Ces travaux ont été initiés dans le cadre d'un programme de recherche du BRGM, sous la coordination de MM. J.P. Chilès et J.P. Sauty. Les auteurs souhaitent souligner la contribution essentielle de M. G. Proust (Ecole des mines de Nancy), et sont redevables à MM. H. Prade et $D$. Dubois (IRIT-Toulouse) de leurs utiles suggestions et avis.

\section{Bibliographie}

Bardossy, A., Bronstert, A., Merz, B., 1995 - I-, 2- and 3-dimensional modeling of groundwater movemement in the unsaturated soil matrix using a fuzzy approach. Advances in Water Resources, vol. 18, n 4 , pp.237-251.

Cleary, R. W., Ungs, M. J., 1978 Analytical models for groundwater pollution and hydrology. Princeton University, Water Resources Program Report 78 WR-15, U.S.A

Collectif, Club Crin « Logique floue », 1997 - Évaluation subjective : méthodes, applications et enjeux. Cahiers des Clubs Crin, Association Ecrin, Paris.

Commission Européenne, 1994. Règlement de la Commission $n^{\circ}$ 1488/94/CE du 28 juin 1994, établissant les principes d'évaluation des risques pour l'homme et pour l'environnement présentés par les substances existantes. JOCE ${ }^{\circ} \mathrm{L} 161 \mathrm{du}$ 29 juin 1994, Luxembourg.

Commission Européenne, 1996. Technical guidance document in support of Commission Directive 93/67/EEC on risk assessment for new notified substances and Commission Regulation (EC) $n^{\circ}$ 1488/94 on risk assessment for existing substances. Parts I to 4. Office des Publications Officielles des Communautés Européennes, Luxembourg.

Dou C., Woldt W., Bogardi I., Dahab M., 1995 - Steady-state groundwater simulation with imprecise parameters. Water Resource Research, Vol. 31, $n^{\circ}$ II, pp. 2709-2719.

Freeze, R.A., Cherry, J., 1979 Groundwater. Prentice Hall (eds.), N.J. U.S.A

Kindler J., Nawalany M., Rogowski R. Tyszewski S., 1988 - Imprecision in groundwater modelling and management. Proceedings of the International Conference on Groundwater Management : Use of models in decision-making, Amsterdam, October 1987, pp. 139-148, Kluwer Academic Publishers.

Prade H., Dubois D., 1988 - Théorie des possibilités - Application à la représentation des connaissances en informatique. Collection Méthode et Programmes, Masson Ed., Paris.

Shaw W., 1989 - Investigations of the potential of fuzzy sets and related approaches for treating uncertainties in radionuclide transfer predictions. EC Report $n^{\circ}$ EUR 12499, Luxembourg. Vose D., 1996 - Quantitative risk analysis - A guide to Monte-Carlo simulation modelling. John Wiley \& Sons Ltd.

Zadeh L., 1965 - Fuzzy Sets - Information and Control, $n^{\circ} 8$, pp. 338-353. 